\title{
Safe and private pedestrian detection by a low-cost fiber-optic specklegram
}

\author{
A. Rodriguez-Cuevas ${ }^{1 *}$, L. Rodriguez-Cobo ${ }^{1,2}$, M. Lomer ${ }^{1,2}$ and J.M. Lopez-Higuera ${ }^{1,2}$ \\ ${ }^{1}$ Photonics Engineering Group, University of Cantabria, 39005, Santander (Spain) \\ ${ }^{2}$ CIBER-bbn, Instituto de Salud Carlos III, 28029, Madrid (Spain)
}

\begin{abstract}
Surveillance is becoming more and more important in the recent years. In many cities, cameras have been set to look after parks, streets, roads, facilities and so on, however this fact is rising concerns about privacy. In this work, an alternative surveillance method which gather at once security and privacy has been propose and tested. Based on fiber optic specklegram technology; a system consisting of a fiber optic, a coherent light source and a photodetector has been placed under a carpet for detecting people walking over it and its accuracy, regarding measuring the steps, have been measured. Results suggest that using low exposed geometries along the carpet and basic processing methods, it is possible to detect with more than $95 \%$ of accuracy the number of steps done by the person walking over the carpet.
\end{abstract}

Keywords: Fiber optic, speckle, monitoring, pedestrian, surveillance, privacy, low cost

\section{INTRODUCTION}

The safety demands from the citizens to avoid all kind of threats have generated an intense grown in the number surveillance systems installed worldwide. However, these surveillance systems frequently conflict with privacy. In many cities cameras have been set to look after parks, streets, roads, facilities and so on, moreover it is highly probable that we will see the number of them increasing all around the world in the next years [1].

Among surveillance cases; pedestrian detection it is a necessity not only in the safety and security field, such as automotive safety or surveillance of areas and facilities, but also in human machine interfaces, and robotics. So far many different systems have been developed and established for that purpose [2]. Cameras are the most extended one, since they are cheap and very useful to get a great amount of information on real time. However, there are some locations in which a surveillance video-camera is not a plausible alternative [3]. In some areas, where the expectation or even the legitimate demand of privacy is required, the pedestrian detection systems can be still required, thus, there is a need of looking for alternative and reliable technologies. In this non-conventional context, low-cost fiber-optic specklegram arise as a suitable technology for pedestrian detection, due to the privacy that the system has indeed, and due to the possibility of sense wide areas.

The speckle phenomenon produced in fiber optics has been studied by many authors, and for rather different applications [4-6]. Spillman et al. defined this phenomenon as an interference between different modes together with a self-mode interaction which is given when a coherent light is propagated through a multimode fiber optic. The output speckle pattern projected by the end of the fiber (specklegram) is composed of a large number of individual speckles (bright dots and dark areas). The intensity of each individual speckle may vary while the total intensity of the speckle pattern must remain constant [5]. Perturbations applied to multimode optical fibers have influence on phase, polarization and distribution of the modes, resulting in visible changes in the speckle pattern. By analyzing these changes within the speckle pattern the original perturbations can be sense.

In this work, the specklegram technology was considered as a possible method to monitor pedestrians. The main point on the research was to develop an optimal combination of geometry distribution, type of fiber and processing method in order to obtain an accurate measurement of the number of steps when someone walking on the monitored carpet. This measurement has been done based on a proof-of-concept system while its accuracy has been obtained to verify the initial hypothesis: whether this technique could be used or not as a surveillance system.

*alberto.rodriguez@unican.es; phone +34 942200877; fax +34 942200877

25th International Conference on Optical Fiber Sensors, edited by Youngjoo Chung, Wei Jin,

Byoungho Lee, John Canning, Kentaro Nakamura, Libo Yuan, Proc. of SPIE Vol. 10323,

103236F · @ 2017 SPIE · CCC code: 0277-786X/17/\$18 · doi: 10.1117/12.2263440 


\section{TEST SEQUENCE AND SET UP}

The first step in this research was to establish some initial conditions to procced with the main tests. Initially some random geometries were tested along the fiber using different lengths of fiber-optic segments, and different diameter core. Following the achieved results, it was decided to use plastic fiber optics with diameter core equal or bigger than 50 micrometers, and lengths of 3.7 meters. In addition, all the fibers selected had an external protection.

After the initial experiments, four different diameter core were tested: 50, 250, 1000, and 2000 micrometers. The four segments were 3.7 meters long, and each of them were tested in three different geometries and using two different photodetector systems: a CCD camera and a photodiode. For every fiber-detector combination, two people walked over the carpet performing six steps. This carpet was 4.3 meters long and 70 centimeters wide. A schematic representation is depicted in Figure 1.
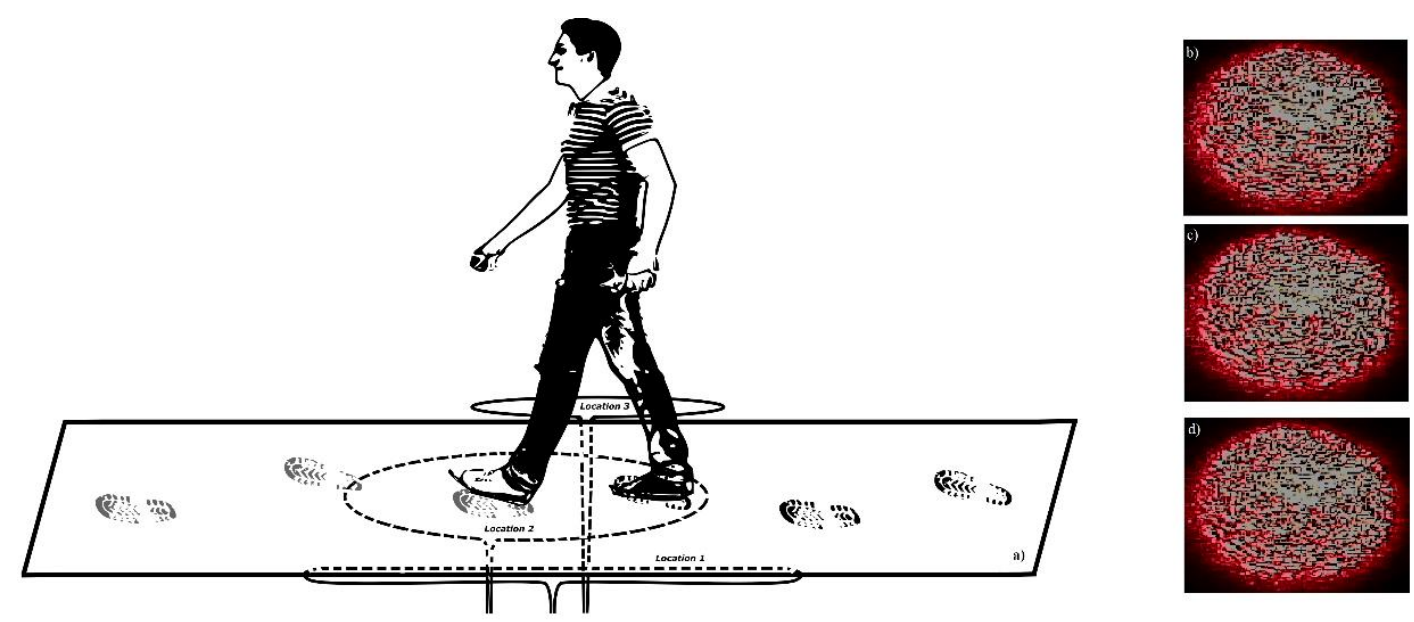

Figure 1. (a) Fiber optic location diagram. Location 1: Parallel position having only one side of the fiber under the carpet. Location 2: Central circle being almost all the fiber length under the carpet. Location 3: Transversal positioning being only the initial and final segments of the fiber under the carpet. (b) Frame 50 of one test captured by the CCD. (c) Frame 51 of one test captured by the CCD. (c) Frame 52 of one test captured by the CCD.

Since the capturing rate and the available data were different in the photodiode and in the CCD camera; two different processing methods were developed: one for the CCD camera and another for the photodiode. Both follow the same processing steps: differential processing, mobile average filter and peak detection.

The differential processing method in the case of the CCD camera was done by subtracting in every pixel the value given in the frame n-1 from the value given in the frame $n$, and afterwards adding all this values of each pixel without taking into consideration their positive or negative sing. Eventually one final value was obtained for each frame, which represents the variation in the speckle pattern in the current frame in reference with the immediately previous one. On the other hand, the differential processing method was done just by subtracting the intensity value from the previous one.

Both the moving average filter and the peak detection function were, indeed, the same in both: CCD and photodiode, with the only exception of the number of values taken for the mobile average. This can be explained due to the fact that the capture rate was different so the faster it was, the more values must be taken for the filter to be accurate. Figure 2 shows the processing sequence in one of the test carried out for both photodiode and CCD camera. Each combination was tested three times by two different people, in order to obtain better statistically outcomes. 

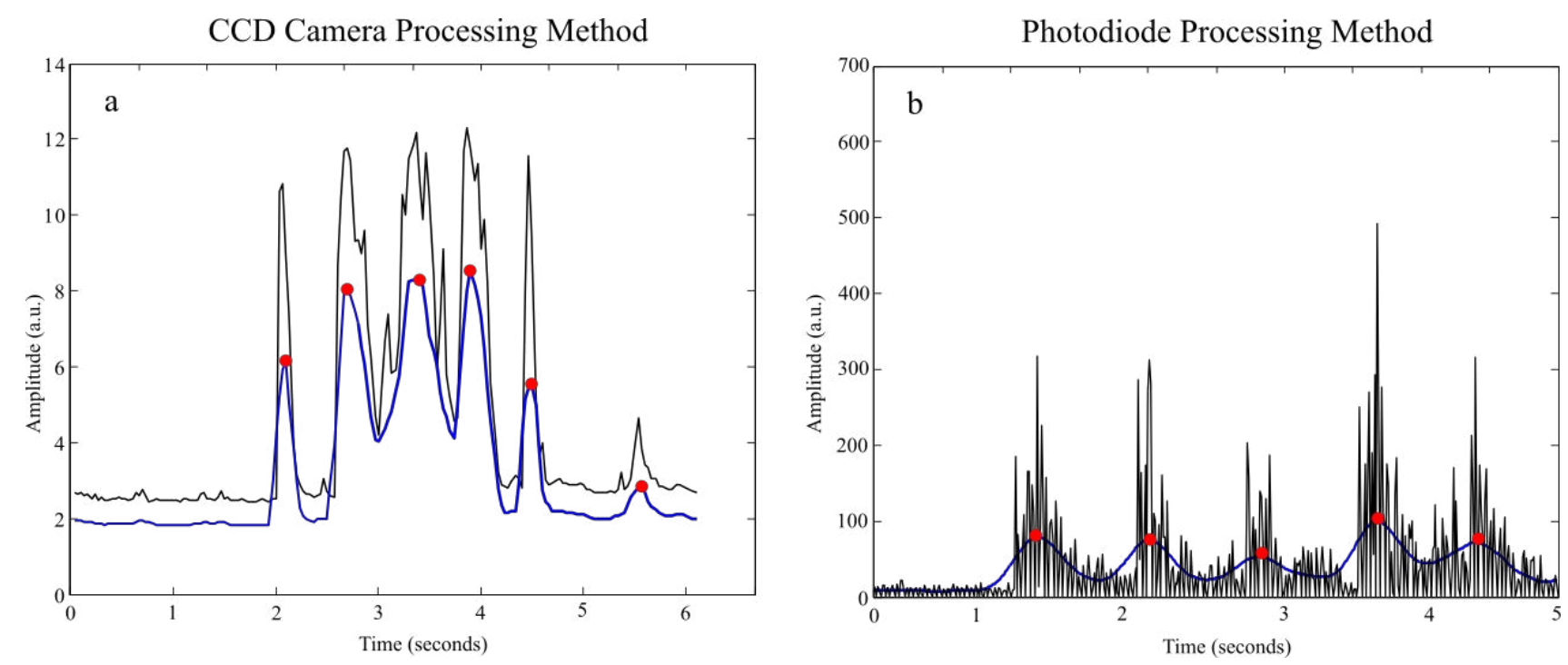

Figure 2. Processing methods: the differential sequence is low-pass filtered before detecting the peaks. (a) Processing method for the CCD camera capture. (b) Processing method for the Photodiode Capture.

\section{RESULTS AND DISCUSSION}

Each accuracy value has been calculated using a trivial equation (Eq. 1). Being $\eta$ the resulting accuracy given in percentage, $\mathrm{X}$ the number of steps detected by the system, $\mathrm{Y}$ the number of steps done by each person, and $\mathrm{n}$ the number of test performed (six for every combination). In Table 1, the achieved results have been summarized.

$$
\eta=\frac{1}{n}\left[\sum_{1}^{n} \frac{X}{Y}\right] * 100
$$

\begin{tabular}{|l|c|c|c|c|c|c|}
\hline \multirow{2}{*}{\begin{tabular}{c}
\multirow{2}{*}{$\begin{array}{c}\text { Fiber Optic } \\
\text { Diameter }\end{array}$} \\
\cline { 2 - 8 }
\end{tabular}} & \multicolumn{3}{|c|}{ CCD Camera } & \multicolumn{4}{c|}{ Photodiode } \\
\cline { 2 - 8 } & Transversal & Parallel & Central circle & Transversal & Parallel & Central circle \\
\hline 50 micrometers & $66.67 \%$ & $44.44 \%$ & $61.11 \%$ & $16.67 \%$ & $8.33 \%$ & $16.67 \%$ \\
\hline 250 micrometers & $72.22 \%$ & $88.89 \%$ & $88.89 \%$ & $55.56 \%$ & $76.39 \%$ & $62.5 \%$ \\
\hline 1000 micrometers & $79.17 \%$ & $94.44 \%$ & $75 \%$ & $75 \%$ & $97.22 \%$ & $91.67 \%$ \\
\hline 2000 micrometers & $69.44 \%$ & $86.11 \%$ & $72.22 \%$ & $84.72 \%$ & $72.22 \%$ & $91.67 \%$ \\
\hline
\end{tabular}

Table 1: Accuracy percentage for all the tested combinations.

The experimental results, suggest that the less exposed geometry (the parallel location) tend to be more accurate than the other locations that pedestrian set foot on it. This fact was confirmed in further in-vivo experiments; in these experiments, whenever the pedestrian stepped on the fiber optic, the generated perturbation was so intense that the speckle pattern had no time to become into a stable state, and thereby, this step and the next one were detected as one single step in the processing method.

In terms of core diameter, the 1000-micrometers fiber optic performs the best of them, while the 50-micrometers fiber rendered useless. This phenomenon suggests that there is a tradeoff between the amount of light delivered to the photodetector system (the more the better), and the number of modes contained by the fiber (related to the diameter of the 
fiber), thus a diameter core bigger than 1000 micrometers is too sensitive. Thereby, when this extra sensitivity is present, each step generates switching states in the speckle pattern too long between one step and the next one, detecting a single step instead of two.

\section{CONCLUSIONS}

Pedestrian detection system based on specklegram technology has been proved as a very accurate system; exhibiting accuracies of $97 \%$ in some cases. Therefore, this technology could be positioned in some scenarios as an alternative to the predominant surveillance-methods (e.g. video camera monitoring), with the advantages discussed in the introduction section: a higher degree of privacy for the people being monitoring and the possibility of sensing wide areas.

When it comes to optimize the sensing process; the main obtained conclusion is that fiber optic should be placed in a way that people walking never set foot on it. It has been observed a wide range of miscalculation in those measurements where the fiber optic had a higher risk of being step over by the person walking, this is, in the central ring location and in the straight configuration; though to a less extender degree. Other method to avoid this problem, could be a better integration or covering for the employed optical fiber.

In terms of diameter, it has been observed that all the plastic fiber optic used instead of the 50 micrometers of diameter core exhibit good performance when measuring steps. Based on the achieved results, it can be concluded that optical fiber must have at least a critical number of modes to exhibit enough sensitivity. Finally, the comparison between CCD camera and photodiode, both performed well in the same locations for diameter core larger than 50 micrometers. The lower information that the photodiode gets from the behavior of each mode is compensated by the faster rate of capture, while, for the 50 micrometers fiber, the intensity received is only enough to perform a CCD detection. The achieved results enable this technology to be employed in real security systems.

\section{ACKNOWLEDGEMENTS}

This work has been supported by the projects TEC2013-47264-C2-1-R and TEC2016-76021-C2-2-R of the Spanish government and by a Parliament of Cantabria postdoc grant.

\section{REFERENCES}

[1] Räty TD., "Survey on contemporary remote surveillance systems for public safety" IEEE Transactions on Systems, Man, and Cybernetics, Part C (Applications and Reviews), 40(5):493-515 (2010).

[2] Enzweiler, M., \& Gavrila, D. M., "Monocular pedestrian detection: Survey and experiments" IEEE Transactions on Pattern Analysis and Machine Intelligence, 31(12), 2179-2195 (2009).

[3] Vagts, H., \& Beyerer, J., "Security and privacy challenges in modern surveillance systems" Proceedings of the Future Security Research Conference, pp. 94-116 (2009).

[4] Lomer M, Rodriguez-Cobo L, Revilla P, Herrero G, Madruga F, Lopez-Higuera JM.. "Speckle POF sensor for detecting vital signs of patients." In OFS2014 23rd International Conference on Optical Fiber Sensors, 91572I91572I. International Society for Optics and Photonics, (2014).

[5] Spillman Jr, W., et al., "Statistical-mode sensor for fiber optic vibration sensing uses" Applied optics, 28(15): p. 3166-3176 (1989).

[6] Yin S, Purwosumarto P, Francis T., "Application of fiber specklegram sensor to fine angular alignment" Opt Commun, 170(1):15-21 (1999). 